\title{
Selected Bibliography on Wang Wei's works
}

Chen Tiemin 陳鐵民. Wang Wei ji jiao zhu 王維集校注. Beijing: Zhonghua shuju, 1997.

. Wang Wei lun gao 王維論稿. Beijing: Renmin wenxue chubanshe, 2006.

.Wang Wei xin lun. 王維新諭. Beijing: Beijing shifan xueyuan chubanshe, 1990.

Chou, Shan. "Beginning with Images in the Nature Poetry of Wang Wei." HJAS 42.1 (1982): 117-37.

Hsieh, Daniel. "'The Nine Songs,' and the Structure of the 'Wang River Collection." Chinese Literature: Essays, Articles, Reviews 35 (2013): $1-30$.

Iritani Sensuke 入谷仙介. $\bar{O} I$ kenkyū 王維研究. Sōbunsha, 1976.

Li Liangwei 李亮偉. Han yong da ya: Wang Wei yu zhongguo wenhua 涵泳大雅：王維與中國文化. Beijing: Zhonghua shuju, 2003.

Liu Shengjun 柳晟俊. Wang Wei shi yanjiu 王維詩研究. Taipei: Liming wenhua shiye gongsi, 1987.

Liu Weichong 劉維崇. Wang Wei ping zhuan 王維評傳. Taibei: Zhengzhong shuju, 1972.

Owen, Stephen. "The Formation of the Tang Estate Poem." HJAS 55:1 (1995): 39-59.

. The Great Age of Chinese Poetry: The High T'ang. New Haven: Yale University Press, 1981.

Pi Shumin 皮述民. Wang Wei tan lun 王維探諭. Taibei: Lianjing chuban shiye gongsi, 1999.

Tan Zhaoyan 譚朝炎. Hong chen fo dao mi wangchuan: Wang Wei de zhutixing quanshi 紅應佛道䚁輞川: 王維的主體性詮释. Beijing: Zhongguo shehui kexue chubanshe, 2004.

Tsuru Haruo 都留春雄, Yoshikawa Kōjirō 吉川幸次郎, Ogawa Tamaki 小川環樹, et al. $\bar{O} I$ 王維. Iwanami Shoten, 1958.

Wagner, Marsha L. Wang Wei. Boston: Twayne Publishers, 1981.

Wang Congren 王從仁. Wang Wei he Meng Haoran 王維和孟浩然. Shanghai: Shanghai guji chubanshe, 1983.

Wang Wei yanjiu 王維研究. Beijing: Zhongguo gongren chubanshe, 1992. 
Warner, Ding Xiang. "The Two Voices of Wangchuan Ji: Poetic Exchange between Wang Wei and Pei Di." Early Medieval China 1011.2 (2005): 57-72.

Yang Jingqing. The Chan Interpretation of Wang Weis Poetry: A Critical Review. Hong Kong: The Chinese University Press, 2007.

Yang Wenxiong 楊文雄. Shifo Wang Wei yanjiu 詩佛王維研究. Taipei: Wen shi zhe chubanshe 1988.

Yu, Pauline. The Poetry of Wang Wei: New Translations and Commentary. Bloomington: Indiana University Press, 1980.

Zhang Qinghua 張清華. Wang Wei nianpu 王維年譜. Shanghai: Xuelin chubanshe, 1989.

Zhuang Shen 莊申. Wang Wei yanjiu 王維研究. Hong Kong: Wanyou tushu gongsi, 1971. 